\title{
Use of lipid parameters to identify apparently healthy men at high risk of arterial stiffness progression
}

\author{
Yu Sang, Ming Cao, Xiaofen Wu, Lei Ruan* and Cuntai Zhang*
}

\begin{abstract}
Background: Dyslipidemia contributes to the development and progression of arterial stiffness. We aimed to identify the most informative measures of serum lipids and their calculated ratios in terms of arterial stiffness progression risk.

Methods: Total cholesterol (TC), triglyceride (TG), low-density lipoprotein cholesterol (LDL-C), high-density lipoprotein cholesterol (HDL-C), and brachial-ankle pulse wave velocity (baPWV) of 659 healthy males (47.4 10.7 years) were measured at baseline. Values for non-HDL-C, TC/HDL-C, TG/HDL-C, LDL-C/HDL-C, and non-HDL-C/HDL-C were calculated. BaPWV was re-performed after 4.1 years follow-up. Elevated baPWV was defined as baPWV $\geq 1400 \mathrm{~cm} / \mathrm{s}$.

Results: Over the follow-up period, the mean baPWV value increased from $1340 \mathrm{~cm} / \mathrm{s}$ to $1410 \mathrm{~cm} / \mathrm{s}$, and 331 individuals increased/persisted with high baPWV (outcome 1). Among the 448 subjects who had normal baseline baPWV, 100 incident elevated baPWV occurred (outcome 2). Only baseline logTG (OR 1.64 [95\% Cl: 1.14-2.37] for outcome $1 ; 1.89$ [1.14-3.17] for outcome 2) and logTG/HDL-C (1.54 [1.15-2.10] for outcome 1; 1.60 [1.05-2.45] for outcome 2) were significantly associated with arterial stiffness progression after adjusting for confounding factors. Adding logTG or logTG/HDL-C to age and blood pressure improved the accuracy of risk predictions for arterial stiffness progression. These associations remained significant when lipids were analyzed as categorical variables.

Conclusions: Baseline serum TG and TG/HDL-C were independently associated with increases in/persistently high baPWV and incident elevated baPWV, and they performed more effectively than other lipid variables in identifying healthy men at high risk of arterial stiffness progression.
\end{abstract}

Keywords: Lipids, Triglyceride, TG/HDL-C, Arterial stiffness, Pulse wave velocity

\section{Background}

Cardiovascular disease (CVD) has become the leading cause of death in China [1]. The increase in CVD risk is largely driven by adverse changes of the vasculature, including arterial stiffening. Brachial-ankle pulse wave velocity (baPWV) is the most widely used routine clinical practice in Asia to assess arterial stiffness and is being increasingly incorporated into studies in the US and Europe [2,3], since the measurement is valid,

*Correspondence: ruanlei8863@sina.com; ctzhang0425@163.com Department of Geriatrics, Tongji Hospital of Tongji Medical College, Huazhong University of Science and Technology, Wuhan, China reproducible, minimal-risk, convenient, and cost-saving. Accumulating evidence demonstrates that arterial stiffness, evaluated by baPWV, is an independent predictor for CVD events and mortality in the general population and various patient populations [4-6]. Arterial stiffness is also one of the major age-related arterial phenotypes [7], and the 'Vascular Aging Continuum' has regarded increased arterial stiffness as the fundamental and vital link [8]. Pulse wave velocity is considered a physiological method for quantifying vascular aging $[9,10]$. Therefore, a relatively simple means to identify individuals at high risk of arterial stiffness progression would be clinically useful. 
Dyslipidemia characterized by the increase of total cholesterol (TC), triglyceride (TG), or low-density lipoprotein cholesterol (LDL-C), or the decrease of highdensity lipoprotein cholesterol (HDL-C) contributes to arterial stiffness and has been proven to be associated with baPWV in extensive cross-sectional studies [7, 11]. However, associations observed in cross-sectional analyses limit inferences about temporality and longitudinal studies regarding the role of serum lipids on arterial stiffness progression are limited and far from conclusive. In addition, arterial stiffness progresses at different rates and can be accelerated by several long-standing cardiovascular risk factors [12], and participants enrolled in previous longitudinal studies were often affected by a variety of chronic diseases. Therefore we selected apparently healthy individuals to reduce the potential bias from other diseases and capture the dyslipidemia-related atrial stiffness progression difference. We aimed to determine the association of baPWV progression with baseline serum lipids, including TC, TG, LDL-C, HDL-C, nonHDL-C, TC/HDL-C, TG/HDL-C, LDL-C/HDL-C, and non-HDL-C/HDL-C, and to confirm the most informative one of those lipid parameters in identifying healthy men at high risk of arterial stiffness progression.

\section{Methods}

\section{Subjects}

This retrospective longitudinal study was conducted at the physical examination center of the geriatric department of Tongji Hospital. The center is not confined to the elderly and is mainly responsible for employee health screening of local enterprises. The study was approved by the medical ethics committee of Tongji Hospital (TJIRB20190410) and the study protocol conforms to the Declaration of Helsinki. Considering the small sample size of women in our center and the sex difference in arterial stiffness [13], we screened the 10,519 baPWV database records of males from March 2011 to July 2019. A total of 1228 individuals had their second baPWV measurement after a delay of more than three years. The exclusion criteria were: age $<18$ years, inheritable dyslipidemia, use of lipid-lowering medications, moderate or server hypertension [defined as systolic blood pressure $(\mathrm{SBP}) \geq 150 \mathrm{~mm} \mathrm{Hg}$, diastolic blood pressure (DBP) $\geq 95 \mathrm{~mm} \mathrm{Hg}$, or use of antihypertensive drugs], diabetes [defined as fasting blood glucose (FBG) of $\geq 7.0 \mathrm{mmol} / \mathrm{L}, \mathrm{HbA} 1 \mathrm{c} \geq 6.5 \%$, or use of diabetes medications], coronary heart disease, stroke, obvious arrhythmia (persistent atrial fibrillation, frequent premature beats, or wearing a pacemaker), cardiomyopathy, valvular heart disease, chronic liver or kidney disease, cancer, anklebrachial index (ABI) less than 0.9 [14], and missing data. Finally, 659 subjects were included in the analysis (Fig. 1).

\section{Baseline clinical characteristics}

Standardized in-person interviews were conducted by trained staff to collect information regarding age, sex, current cigarette smoking status, medical history, and medication use. Anthropometric indexes including height and weight were measured. Body mass index (BMI) was computed as the weight in kilograms divided by the square of the height in meters. Blood pressure and heart rate were measured using an OMRON sphygmomanometer (OMRON Corporation, Japan). The blood pressure and heart rate used in the analysis were calculated as the average of three measured values. Mean arterial pressure (MAP) was calculated from the standard equation $\mathrm{MAP}=(2 / 3) \mathrm{DBP}+(1 / 3) \mathrm{SBP}$ (in $\mathrm{mm} \mathrm{Hg}$ ). Fasting venous blood samples were collected and sent to the hospital's clinical chemistry laboratory. TC, TG, LDL-C, HDL-C, FBG, HbA1c, creatinine, and uric acid were measured using standard certified assays. We also calculated values for non-HDL-C (TC minus HDL-C), TC/HDL-C (TC divided by HDL-C), TG/HDL-C (TG divided by HDL-C), LDL-C/HDL-C (LDL-C divided by HDL-C), and non-HDL-C/HDL-C (non-HDL-C divided by HDL-C).

\section{Arterial stiffness measurements at baseline and follow-up and definition of the outcomes}

BaPWV and ABI were measured using the Vascular Profiler BP-203RPEIII system (Omron, Kyoto, Japan). Trained technicians placed the pressure cuffs on the subjects, i.e., one on the upper part of each arm and one on each ankle. Then, the subjects were examined after ten minutes of rest in the supine position. The device simultaneously recorded the bilateral pulse waves of the brachial and posterior tibial arteries using an oscillometric method. BaPWV was calculated as the ratio of the traveled distance (which was automatically estimated from the body height) divided by the transit time of the pulse wave between the brachial and posterior tibial arteries. We classified the outcome in two different manners. Outcome 1: the baseline and follow-up baPWV were both divided into quartiles, respectively. Then we classified subjects into two subgroups: those who decreased their quartile distribution or persisted within the two lower quartile groups, and those who increased their quartile distribution or persisted within the two higher quartile groups [15]. Outcome 2: a cutoff value of more than $1400 \mathrm{~cm} / \mathrm{s}$ for baPWV was used to diagnose elevated baPWV [4, 16-18]. Individuals with normal baseline baPWV were divided into nonelevated baPWV and incident elevated baPWV groups based on their follow-up baPWV levels. 


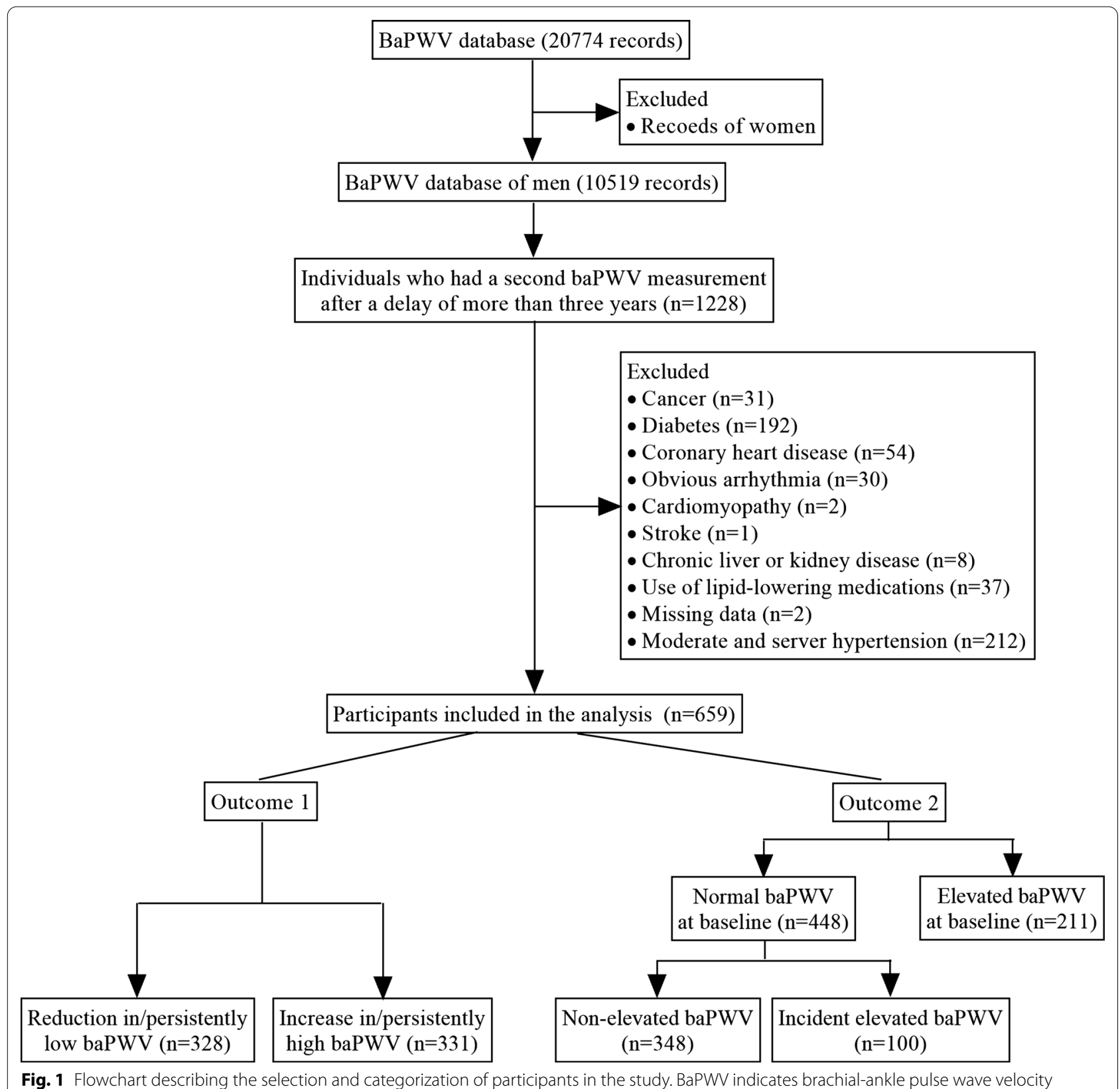

\section{Statistical analysis}

Data were analyzed using $\mathrm{R}$ and RStudio 3.6.2. Continuous variables were presented as the means \pm standard deviation or medians (interquartile range), as appropriate for the distribution. Categorical variables were shown as counts and proportions. Paired t-tests were used to determine if the baPWV levels changed over the followup period. We compared the baseline variables between groups using unpaired t-test, Mann-Whitney $\mathrm{U}$ test, and Chi-squared test accordingly. For comparison, identical crude and multivariable-adjusted binary logistic regression models were built for each lipid parameter respectively. Odds ratios (ORs) and $95 \%$ confidence intervals (CIs) for the two outcomes were calculated. Prior to regression analysis, TG and TG/HDL-C were log-transformed to achieve normality. Age and MAP, the most important determinants for baPWV, were controlled for in the first model. Further adjustments were made for BMI, current smoking status, heart rate, FBG, creatinine, and uric acid in the second model. All the covariates included were measured at baseline. The improvement in the ability of each serum lipid to predict outcomes 
was summarized using area under the receiver operating characteristic (ROC) curves (AUC), positive net reclassification improvement (NRI), and integrated discrimination improvement (IDI) [19]. In sensitivity analyses, TC, TG, HDL-C, LDL-C, and non-HDL were divided into three or two levels by clinical cut-points [20]; TC/HDLC, TG/HDL-C, LDL-C/HDL-C, and non-HDL-C/HDL-C ratios were divided into three levels by tertile cut-points. ORs and 95\% CIs for the outcomes across categories of each serum lipid were also calculated, using the most favorable category as the reference. Trends in ORs across categories of each lipid were calculated by modeling the lipid categories as an ordinal variable. Two-tailed $p$-values $<0.05$ were considered significant.

\section{Results}

\section{General characteristics}

The clinical characteristics of the study subjects are shown in Table 1 . The population had a mean baseline age of $47.4 \pm 10.7$ years, ranging from 22 to 94 years. Sixty-seven (10.2\%) individuals were aged 60 years or older, and 6 of them were the oldest-old (aged 80 and older). The median duration of the follow-up was 4.4 years. The mean baPWV value increased from $1340 \pm 209 \mathrm{~cm} / \mathrm{s}$ to $1410 \pm 245 \mathrm{~cm} / \mathrm{s}$ over the follow-up period $(p<0.001)$.

Of all the study participants, 331 (50.2\%) individuals had increased/persisted with high baPWV (outcome 1), and they had higher levels of age, blood pressure, heart rate, ABI, FBG, HbA1c, TG, TC/HDL-C, TG/HDL-C, and non-HDL-C/HDL-C at baseline, compared with those who presented a reduction or persisted with low baPWV. There were no statistical differences in other lipids, including TC, LDL-C, non-HDL-C, HDL-C, and LDL-C/HDL-C.

A total of 448 subjects had normal baseline baPWV, and $100(22.3 \%)$ of them had elevated baPWV at

Table 1 Baseline characteristics of the individuals included in the analysis

\begin{tabular}{|c|c|c|c|c|c|c|c|}
\hline Variables & $\begin{array}{l}\text { Total } \\
(n=659)\end{array}$ & $\begin{array}{l}\text { Reduction in/ } \\
\text { persistently low } \\
\text { baPWV } \\
(n=328)\end{array}$ & $\begin{array}{l}\text { Increase in/ } \\
\text { persistently high } \\
\text { baPWV } \\
(n=331)\end{array}$ & $p$ value $^{a}$ & $\begin{array}{l}\text { Non-elevated baPWV } \\
(n=348)\end{array}$ & $\begin{array}{l}\text { Incident } \\
\text { elevated } \\
\text { baPWV } \\
(n=100)\end{array}$ & $p$ value $^{\mathrm{b}}$ \\
\hline Age, years & $47.4 \pm 10.7$ & $43.4 \pm 8.7$ & $51.5 \pm 10.9$ & $<0.001$ & $43.2 \pm 8.8$ & $49.8 \pm 7.9$ & $<0.001$ \\
\hline Smoker, \% & $241(36.6)$ & $125(38.1)$ & $116(35.0)$ & 0.46 & $140(40.2)$ & $34(34.0)$ & 0.31 \\
\hline $\mathrm{BMI}, \mathrm{kg} / \mathrm{m} 2$ & $24.6 \pm 2.8$ & $24.7 \pm 2.6$ & $24.6 \pm 2.9$ & 0.84 & $24.6 \pm 2.8$ & $24.5 \pm 3.1$ & 0.71 \\
\hline $\mathrm{SBP}, \mathrm{mm} \mathrm{Hg}$ & $121 \pm 11$ & $119 \pm 11$ & $123 \pm 11$ & $<0.001$ & $117 \pm 10$ & $121 \pm 10$ & 0.003 \\
\hline $\mathrm{DBP}, \mathrm{mm} \mathrm{Hg}$ & $75 \pm 9$ & $73 \pm 9$ & $76 \pm 9$ & $<0.001$ & $71 \pm 8$ & $75 \pm 8$ & $<0.001$ \\
\hline MAP, $\mathrm{mm} \mathrm{Hg}$ & $90 \pm 9$ & $89 \pm 9$ & $92 \pm 9$ & $<0.001$ & $87 \pm 8$ & $90 \pm 8$ & $<0.001$ \\
\hline Heart rate, beats/min & $67 \pm 10$ & $66 \pm 10$ & $68 \pm 9$ & 0.008 & $65 \pm 9$ & $66 \pm 8$ & 0.10 \\
\hline$A B \mid$ & $1.10 \pm 0.08$ & $1.09 \pm 0.08$ & $1.11 \pm 0.08$ & 0.005 & $1.09 \pm 0.08$ & $1.11 \pm 0.08$ & 0.03 \\
\hline $\mathrm{FBG}, \mathrm{mmol} / \mathrm{L}$ & $5.04 \pm 0.52$ & $4.99 \pm 0.50$ & $5.08 \pm 0.54$ & 0.03 & $4.97 \pm 0.50$ & $5.08 \pm 0.53$ & 0.06 \\
\hline $\mathrm{HbA} 1 \mathrm{c}, \%$ & $5.58 \pm 0.31$ & $5.55 \pm 0.31$ & $5.61 \pm 0.30$ & 0.02 & $5.54 \pm 0.30$ & $5.61 \pm 0.27$ & 0.05 \\
\hline creatinine, $\mu \mathrm{mol} / \mathrm{L}$ & $81.0 \pm 10.8$ & $81.2 \pm 10.2$ & $80.8 \pm 11.3$ & 0.68 & $81.3 \pm 10.4$ & $80.7 \pm 11.9$ & 0.60 \\
\hline Uric acid, umol/L & $376 \pm 76$ & $376 \pm 76$ & $376 \pm 76$ & 0.94 & $373 \pm 75$ & $385 \pm 80$ & 0.18 \\
\hline $\mathrm{TC}, \mathrm{mmol} / \mathrm{L}$ & $4.74 \pm 0.86$ & $4.74 \pm 0.83$ & $4.74 \pm 0.90$ & 0.97 & $4.71 \pm 0.83$ & $4.78 \pm 0.92$ & 0.50 \\
\hline $\mathrm{TG}, \mathrm{mmol} / \mathrm{L}$ & $1.36(1.01)$ & $1.30(0.83)$ & $1.42(1.09)$ & 0.005 & $1.29(0.80)$ & $1.60(1.08)$ & 0.005 \\
\hline LDL-C, mmol/L & $2.94 \pm 0.74$ & $2.96 \pm 0.69$ & $2.92 \pm 0.78$ & 0.43 & $2.96 \pm 0.72$ & $2.91 \pm 0.81$ & 0.62 \\
\hline Non-HDL-C, mmol/L & $3.56 \pm 0.84$ & $3.54 \pm 0.81$ & $3.59 \pm 0.87$ & 0.52 & $3.52 \pm 0.83$ & $3.61 \pm 0.90$ & 0.39 \\
\hline $\mathrm{HDL}-\mathrm{C}, \mathrm{mmol} / \mathrm{L}$ & $1.18 \pm 0.26$ & $1.20 \pm 0.25$ & $1.16 \pm 0.28$ & 0.06 & $1.19 \pm 0.24$ & $1.17 \pm 0.32$ & 0.60 \\
\hline $\mathrm{TC} / \mathrm{HDL}-\mathrm{C}$ & $4.18 \pm 1.02$ & $4.10 \pm 0.98$ & $4.26 \pm 1.06$ & 0.04 & $4.09 \pm 1.02$ & $4.26 \pm 1.06$ & 0.16 \\
\hline $\mathrm{TG} / \mathrm{HDL}-\mathrm{C}$ & $1.21(1.04)$ & $1.11(0.87)$ & $1.30(1.22)$ & 0.003 & $1.11(0.90)$ & $1.51(1.35)$ & 0.01 \\
\hline LDL-C/HDL-C & $2.59 \pm 0.74$ & $2.56 \pm 0.71$ & $2.61 \pm 0.78$ & 0.32 & $2.56 \pm 0.73$ & $2.61 \pm 0.82$ & 0.65 \\
\hline Non-HDL-C/HDL-C & $3.18 \pm 1.02$ & $3.10 \pm 0.98$ & $3.26 \pm 1.06$ & 0.04 & $3.09 \pm 1.02$ & $3.26 \pm 1.06$ & 0.15 \\
\hline Baseline baPWV, cm/s & $1340 \pm 209$ & $1280 \pm 165$ & $1410 \pm 226$ & $<0.001$ & $1210 \pm 100$ & $1300 \pm 70$ & 0.001 \\
\hline Follow-up baPWV, cm/s & $1410 \pm 245$ & $1250 \pm 112$ & $1570 \pm 240$ & $<0.001$ & $1250 \pm 95$ & $1520 \pm 99$ & 0.001 \\
\hline
\end{tabular}

Data are mean \pm standard deviation, median (interquartile range), or $\mathrm{n}(\%)$

$B M /$ body mass index, $S B P$ systolic blood pressure, $D B P$ diastolic blood pressure, $M A P$ mean arterial pressure, $A B I$ ankle-brachial index, $F B G$ fasting blood glucose, $T C$ total cholesterol, TG triglyceride, $L D L$-C low-density lipoprotein cholesterol, HDL-C high-density lipoprotein cholesterol

a Reduction in/persistently low baPWV versus increase in/persistently high baPWV

b Non-eleated baPWV versus incident elevated baPWV. $p$ values were calculated using unpaired t-test, Mann-Whitney $U$ test, or $\chi^{2}$ test, as appropriate 
follow-up (outcome 2). Individuals with incident elevated baPWV had higher levels of age, blood pressure, ABI, HbA1c, TG, and TG/HDL-C, compared with those who stayed arterial health. Other lipids, including TC, LDL-C, non-HDL-C, HDL-C, TC/HDL-C, LDL-C/ HDL-C, and non-HDL-C/HDL-C had no statistical difference.

\section{Arterial stiffness progression and baseline lipid parameters as continuous variables}

Only baseline $\log$ TG and $\log$ TG/HDL-C were significantly associated with increased risk of arterial stiffness progression in both crude and adjusted logistic regression models (all $p<0.05$; Fig. 2). After adjusting for the baseline confounding factors, a unit increase in baseline $\log$ TG and $\log$ TG/HDL-C resulted in ORs for outcome 1 of 1.64 (95\% CI: 1.14-2.37) and 1.54 (95\% CI: 1.15-2.10), respectively. Meanwhile, the ORs for outcome 2 per unit increase in baseline $\log \mathrm{TG}$ and $\log \mathrm{TG} / \mathrm{HDL}-\mathrm{C}$ was 1.89 (95\% CI: 1.14-3.17) and 1.60 (95\% CI: 1.05-2.45), respectively. TC/HDL-C and non-HDL-C/HDL-C were not significantly associated with arterial stiffness progression in the adjusted models, while the ORs of TC, LDL-C, nonHDL-C, HDL-C, and LDL-C/HDL-C did not reach statistical significance in all the models.

We further performed ROC analyses to study the predictive power of baseline $\log$ TG and $\log$ TG/HDL-C (Fig. 3 and Table 2). When adding logTG or logTG/ HDL-C to the basic model (age + MAP), the prediction of the outcomes showed an increase in AUC. For example, AUC for outcome 1 increased from 0.73 to 0.74 when $\log$ TG was added. Positive NRI and IDI indicated that adding $\log \mathrm{TG}$ or $\log \mathrm{TG} / \mathrm{HDL}-\mathrm{C}$ significantly improved risk reclassification for both outcomes.

\section{Arterial stiffness progression and baseline lipid parameters as categorical variables}

In sensitivity analysis, serum lipids were converted to categorical variables (Fig. 4). TG and TG/HDL-C were significantly associated with both outcome 1 and outcome 2 . The ORs associated with the increased versus appropriate level of TG $(\geq 2.30$ versus $\leq 1.69 \mathrm{mmol} / \mathrm{L})$ was 1.99 (95\% CI: $1.20-3.32$ ) for outcome 1 and 2.33 (95\% CI: 1.12-4.83) for outcome 2. The ORs associated with the highest versus lowest quartile of TG/HDL was 2.04 (95\% CI: $1.27-3.31 ; \geq 1.57$ versus $\leq 0.92$ ) for outcome 1 and 2.77 (95\% CI: $1.37-5.74 ; \geq 1.47$ vs. $\leq 0.87$ ) for outcome 2 .

\section{Discussion}

In this study, we compared the predictive power of baseline serum lipid parameters on arterial stiffness progression in a sample of apparently healthy Chinese men. We found that even after adjusting for the confounding factors, high baseline TG and TG/HDL-C were correlated with high risk of increases in/persistently high baPWV and incident elevated baPWV. In addition, we also found that serum TG was positively associated with baPWV in 794 healthy elderly subjects in a previous study [10]. Hence we confirmed a correlation between TG and arterial stiffness in mid- and late-life.

High TG is a traditional risk factor for CVD [21]. However, previous investigations, which have included TG in multivariable regression analyses for arterial stiffness progression, were conflicting. Some studies showed that baseline TG levels and changes of TG levels over time independently predicted arterial stiffness progression after adjusting for other cardiovascular risk factors [22-25], while others have not [26-29]. TG/HDL-C has been considered as an independent predictor of insulin resistance [30, 31], diabetes mellitus [32], and CVD [33]. High-level TG/HDL-C ratio was also proved to be associated with higher arterial stiffness in cross-sectional studies [34-36], and the relationship might be non-linear [34]. TG/HDL-C was also found to be an independent determinant of arterial stiffness in adolescents and young adults [37]. Consistent with our study, subjects with high TG/HDL-C ratio also had high risk of carotid-femoral pulse wave velocity (cfPWV) progression in healthy individuals [38]. However, the result of another investigation revealed that higher cfPWV in late-life was not related to faster annual rates of change in TG/HDL-C from mid-life [36]. The conflicting results of these investigations were likely based on the differences in the study populations and the evaluation indexes of arterial stiffness and we provided evidence of the independent predictive value of baseline TG and TG/HDL for baPWV progression in Chinese healthy males.

It has long been thought that arterial stiffness is only a feature of hypertension-mediated organ damage, while more recent studies show that arterial stiffness precedes hypertension [39, 40]. Arterial stiffness reflects the cumulative damage of cardiovascular risk factors on the vascular wall and is even better than the blood pressure in reflecting the risk of CVD [41]. Arterial stiffness begins in early childhood and shows increasing progression in adults [7]. Our study population displayed a mean baPWV increase of $16 \mathrm{~cm} / \mathrm{s}$ per year. The progression rate was quite similar to that of another group of Chinese males, in which the 5-year change was $70 \mathrm{~cm} / \mathrm{s}$ for the healthy men [23]. The average annual change of baPWV of a Japanese/American population-based cohort study was $9 \mathrm{~cm} / \mathrm{s}$, but this study merely focused on men aged 40 to 49 years [42]. Valid baPWV progression rates have not been established so far.

China is the world's largest consumer of tobacco, and more than a third of the included individual in our study 


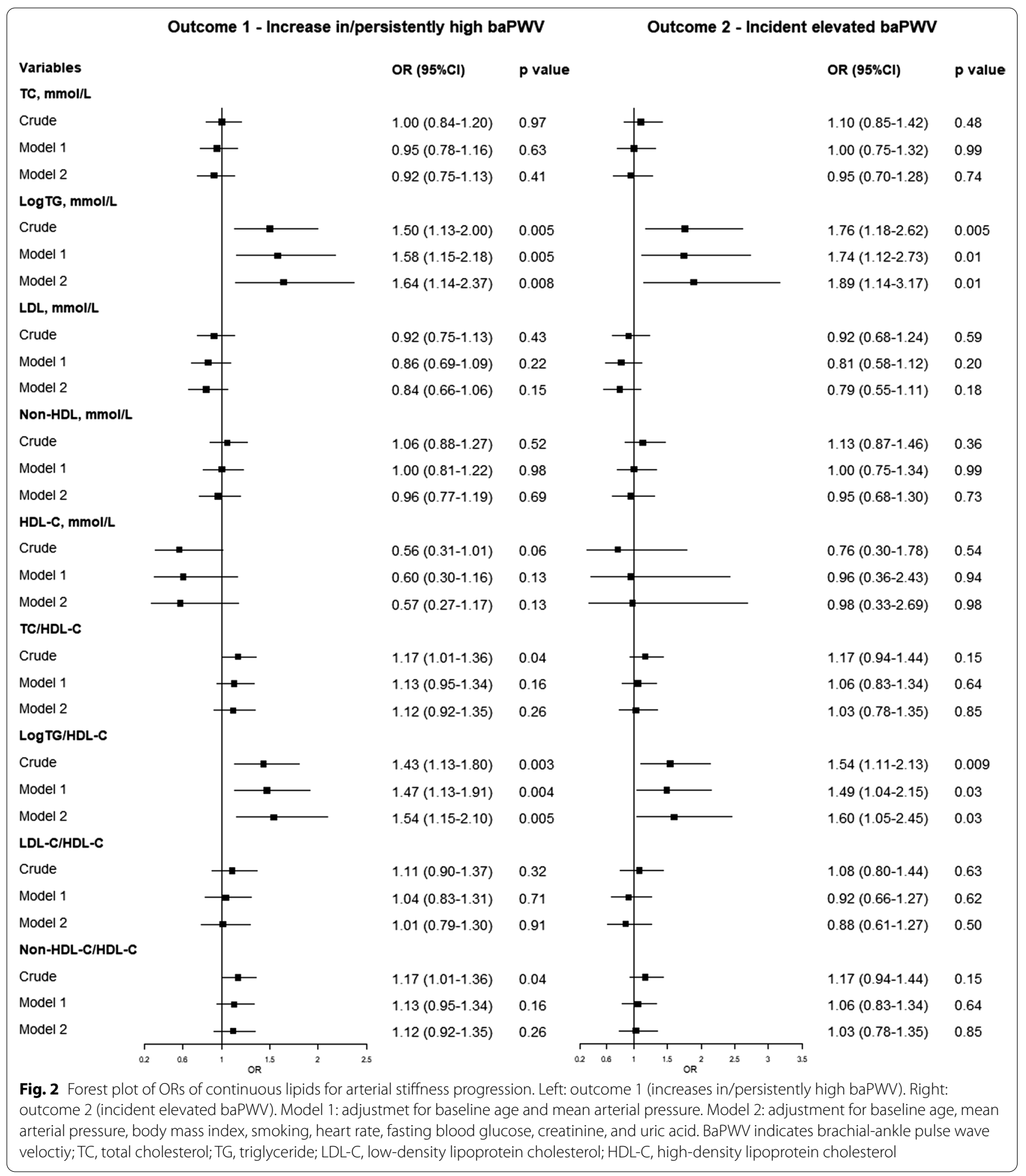

were current smokers at baseline, which was consistent with the finding of national surveys done in China [43]. There is evidence indicating that cigarette smoking can alter the critical enzymes of lipid transport and therefore exerts negative effects on the lipid and lipoprotein profile and [44]. Smoking increases levels of TC, TG, and LDL-C, while decreases the cardio-protective HDL-C [44]. Meanwhile, previous longitudinal studies 
Outcome 1 - Increase in/persistently high baPWV

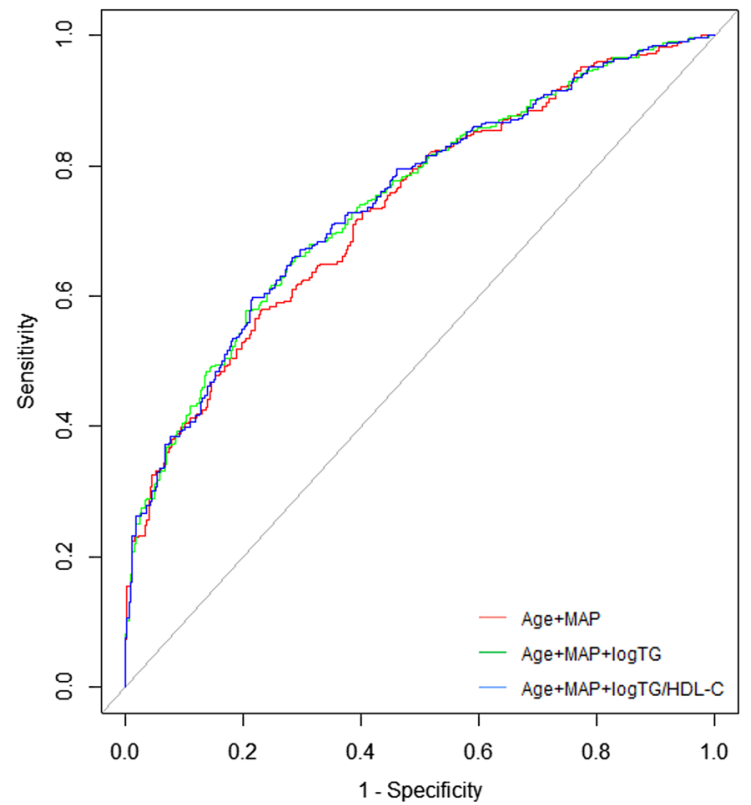

Outcome 2 - Incident elevated baPWV

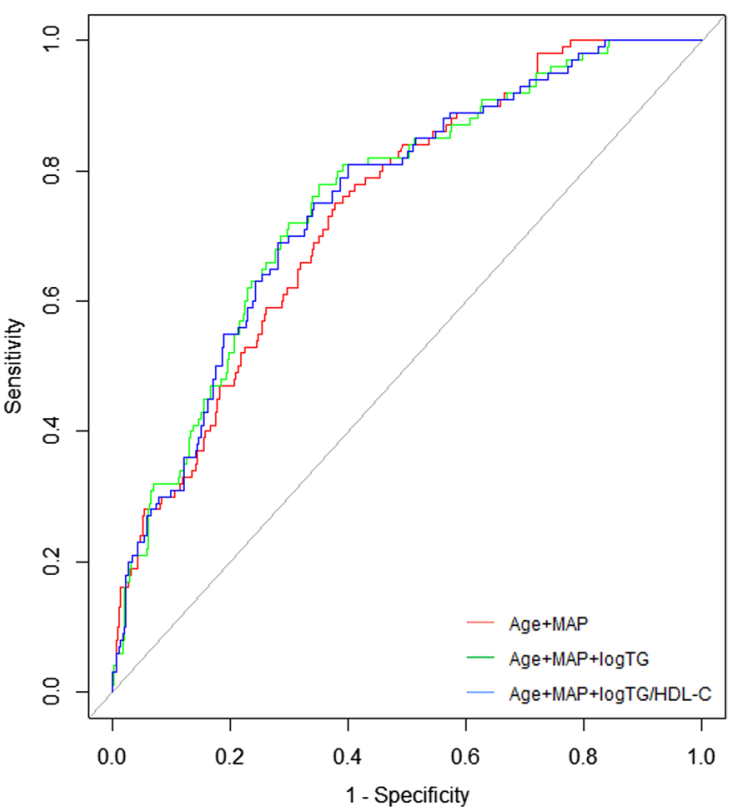

Fig. 3 ROC analyses of logTG and logTG/HDL-C for the prediction of arterial stiffness progression. Left: outcome 1 (increases in/persistently high baPWV). Right: outcome 2 (incident elevated baPWV). BaPWV indicates brachial-ankle pulse wave veloctiy; MAP, mean arterial pressure; TG, triglyceride; HDL-C, high-density lipoprotein cholesterol

Table 2 Model Performance for arterial stiffness progression

\begin{tabular}{|c|c|c|c|c|c|}
\hline Models & $\operatorname{AUC}(95 \% \mathrm{Cl})$ & $\mathrm{NRI}(95 \% \mathrm{Cl})$ & p value & IDI $(95 \% \mathrm{Cl})$ & p value \\
\hline \multicolumn{6}{|c|}{ Outcome 1: Increase in/persistently high baPWV } \\
\hline Age + MAP & $0.73(0.70-0.77)$ & - & - & - & - \\
\hline Age + MAP + LogTG & $0.74(0.71-0.78)$ & $22.9 \%(7.8-37.9 \%)$ & 0.003 & $1.1 \%(0.4-1.9 \%)$ & 0.004 \\
\hline Age + MAP + LogTG/HDL-C & $0.74(0.71-0.78)$ & $22.2 \%(7.0-37.4 \%)$ & 0.004 & $1.2 \%(0.4-2.0 \%)$ & 0.003 \\
\hline \multicolumn{6}{|c|}{ Outcome 2: Incident elevated baPWV } \\
\hline Age + MAP & $0.74(0.69-0.79)$ & - & - & - & - \\
\hline Age + MAP + LogTG & $0.75(0.70-0.81)$ & $40.7 \%(18.8-62.6 \%)$ & $<0.001$ & $1.4 \%(0.1-2.6 \%)$ & 0.03 \\
\hline Age + MAP + LogTG/HDL-C & $0.75(0.70-0.80)$ & $32.1 \%(10.1-54.1 \%)$ & 0.004 & $1.1 \%(0.0-2.2 \%)$ & 0.06 \\
\hline
\end{tabular}

$A U C$ area under the receiver operating curve, $N R I$ net reclassification improvement, IDI integrated discrimination improvement, $M A P$ mean arterial pressure, $T G$ triglyceride, HDL-C high-density lipoprotein cholesterol

have demonstrated that continuous smoking accelerated the arterial stiffness progression in both adolescents and adults even at low levels of smoking exposure [29, 45]. It may be meaningful to study the mediating effects of lipids in the association between smoking and vascular elasticity damage, but there has been very little research into this subject yet. Moreover, detailed data on both time since quitting among past smokers and pack-years among current smokers were not available in the present study, and we did not find a significant relationship between smoking status as a dichotomous yes/no measure and arterial stiffness progression. But we showed that baseline TG and TG/HDL-C were able to predict arterial stiffness progression even after simultaneous adjustment for smoking and other risk factors. Interestingly, studies on the role of lipids in the association between smoking and CVD have been published but far from a convincing conclusion [46-48].

We have some other limitations to consider. First, although the results were adjusted for multiple covariates that may be associated with baPWV, the possibility of residual confounds remains. Second, we referred to some articles about cfPWV, because data regarding baPWV progression is limited. Although baPWV is 


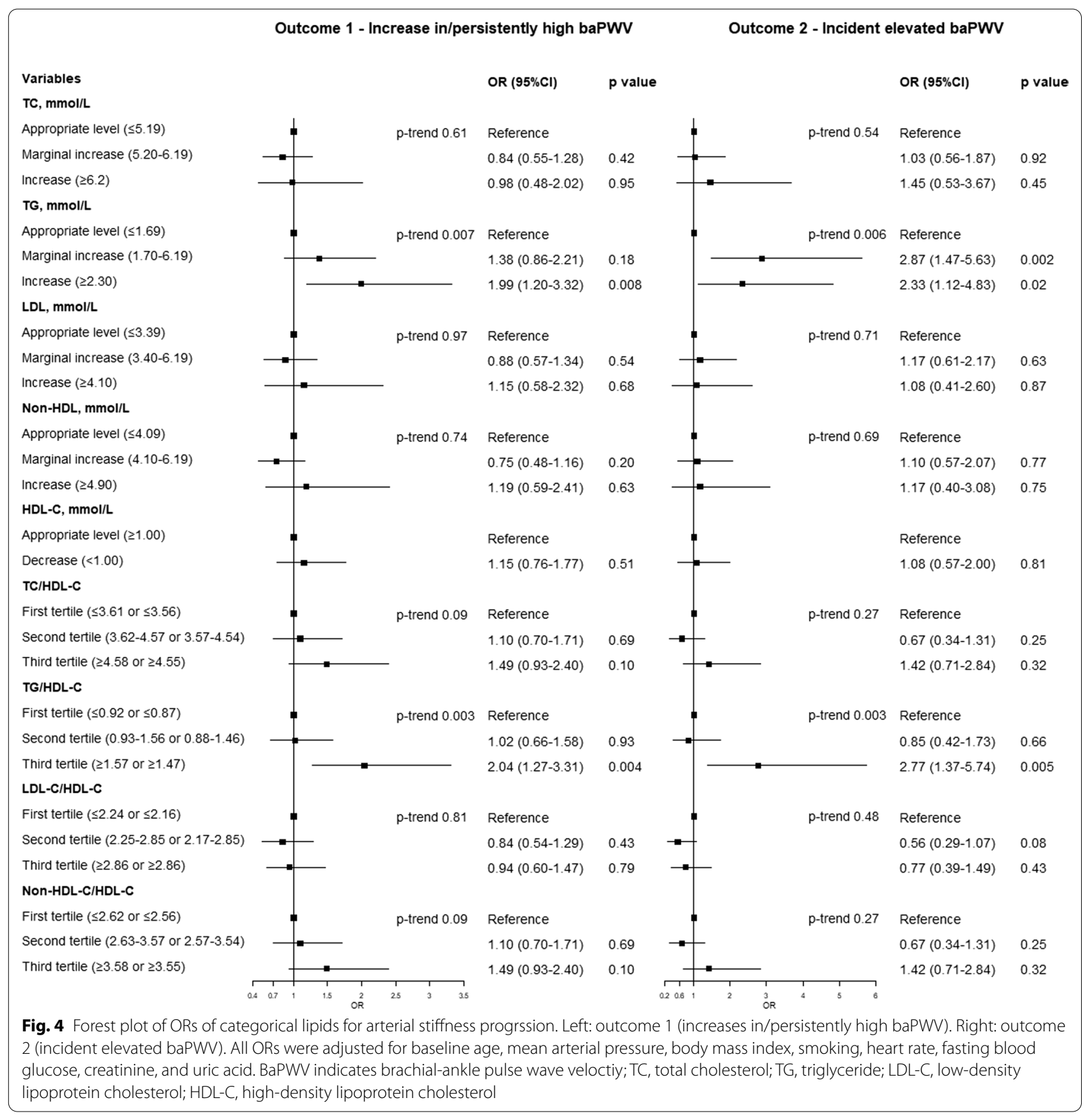

highly correlated with cfPWV, we do not think the results are interchangeable. Third, the results of this study may not be generalizable to women and individuals with comorbidities. Moreover, whether controlling for TG and HDL-C can attenuate progression of arterial stiffness or whether any long-term reduction in arterial stiffness can translate into a reduction in cardiovascular events has not been directly demonstrated in randomized controlled trials.

\section{Conclusions}

In conclusion, we provided evidence in this longitudinal study that baseline TG and TG/HDL-C which could be obtained from routine serum lipids independently predicted increases in/persistently high baPWV and incident elevated baPWV, and they were superior to other traditional lipid variables in identifying healthy men who are at an increased risk of arterial stiffness progression. This positive association between TG, TG/HDL-C, and 
baPWV progression should be considered in the management of vascular health.

\begin{abstract}
Abbreviations
CVD: Cardiovascular disease; BaPWV: Brachial-ankle pulse wave velocity; TC: Total cholesterol; TG: Triglyceride; LDL-C: Low-density lipoprotein cholesterol; HDL-C: High-density lipoprotein cholesterol; SBP: Systolic blood pressure; DBP: Diastolic blood pressure; FBG: Fasting blood glucose; ABI: Ankle-brachial index; BMI: Body mass index; MAP: Mean arterial pressure; OR: Odds ratio; Cl: Confidence interval; ROC: Receiver operating characteristic; AUC: Area under the receiver operating characteristic; NRI: Net reclassification improvement; IDI: Integrated discrimination improvement; CfPWV: Carotid-femoral pulse wave velocity.
\end{abstract}

\section{Acknowledgements}

We appreciate the help and support from all participants who took part in the study and the staff at the physical examination center of the geriatric department of Wuhan Tongji Hospital.

\section{Authors' contributions}

C.Z. and L.R. conceived and designed this study; Y.S. analyzed the patient data and wrote the manuscript; M.C. and X.W. conducted the quality assurance and reviewed and edited the paper. All authors read and approved the final manuscript.

\section{Funding}

This work was supported by the Natural Science Foundation of Hubei Province (Program No. 2017CFB351) and the Major Technology Innovation of Hubei Province (Program No. 2019ACA141).

\section{Availability of data and materials}

The datasets analyzed in the present study are available from the corresponding author on reasonable request.

\section{Ethics approval and consent to participate}

The study was approved by the Ethics Committee of the Tongji Hospital, Tongji Medical College, Huazhong University of Science and Technology (NO: TJ-IRB20190410) in compliance with the Declaration of Helsinki, and all participants provided informed consent.

\section{Consent for publication}

Not applicable.

\section{Competing interests}

The authors declare that they have no competing interests.

Received: 30 September 2020 Accepted: 29 December 2020 Published online: 13 January 2021

\section{References}

1. Zhao D, Liu J, Wang M, Zhang X, Zhou M. Epidemiology of cardiovascular disease in China: current features and implications. Nat Rev Cardiol. 2019;16:203-12.

2. Hollands WJ, Tapp H, Defernez M, Perez Moral N, Winterbone MS, Philo M, et al. Lack of acute or chronic effects of epicatechin-rich and procyanidinrich apple extracts on blood pressure and cardiometabolic biomarkers in adults with moderately elevated blood pressure: a randomized, placebocontrolled crossover trial. Am J Clin Nutr. 2018;108:1006-14.

3. Endes S, Schaffner E, Caviezel S, Dratva J, Autenrieth CS, Wanner M, et al. Physical activity is associated with lower arterial stiffness in older adults: results of the SAPALDIA 3 Cohort Study. Eur J Epidemiol. 2016;31:275-85.

4. Maeda Y, Inoguchi T, Etoh E, Kodama Y, Sasaki S, Sonoda N, et al. Brachialankle pulse wave velocity predicts all-cause mortality and cardiovascular events in patients with diabetes: the Kyushu Prevention Study of Atherosclerosis. Diabetes Care. 2014;37:2383-90.
5. Sheng CS, Li Y, Li LH, Huang QF, Zeng WF, Kang YY, et al. Brachial-ankle pulse wave velocity as a predictor of mortality in elderly Chinese. Hypertension. 2014;64:1124-30.

6. Ohkuma T, Ninomiya T, Tomiyama H, Kario K, Hoshide S, Kita Y, et al. Brachial-ankle pulse wave velocity and the risk prediction of cardiovascular disease: an individual participant data meta-analysis. Hypertension. 2017;69:1045-52.

7. Lu Y, Pechlaner R, Cai J, Yuan H, Huang Z, Yang G, et al. Trajectories of agerelated arterial stiffness in chinese men and women. J Am Coll Cardiol. 2020;75:870-80.

8. O'Rourke MF, Safar ME, Dzau V. The cardiovascular continuum extended: aging effects on the aorta and microvasculature. Vasc Med. 2010;15:461-8.

9. Niiranen TJ, Lyass A, Larson MG, Hamburg NM, Benjamin EJ, Mitchell GF, et al. Prevalence, correlates, and prognosis of healthy vascular aging in a western community-dwelling cohort: the Framingham heart study. Hypertension. 2017;70:267-74.

10. Sang Y, Wu X, Miao J, Cao M, Ruan L, Zhang C. Determinants of BrachialAnkle pulse wave velocity and vascular aging in healthy older subjects. Med Sci Monit. 2020;26:e923112.

11. Zhan B, Huang X, Wang J, Qin X, Zhang J, Cao J, et al. Association between lipid profiles and arterial stiffness in Chinese patients with hypertension: insights from the CSPPT. 2019;70:515-522.

12. Laurent S, Cockcroft J, Van Bortel L, Boutouyrie P, Giannattasio C, Hayoz $D$, et al. Expert consensus document on arterial stiffness: methodological issues and clinical applications. Eur Heart J. 2006;27:2588-605.

13. DuPont JJ, Kenney RM, Patel AR, Jaffe IZ. Sex differences in mechanisms of arterial stiffness. Br J Pharmacol. 2019;176:4208-25.

14. Ato D. Pitfalls in the ankle-brachial index and brachial-ankle pulse wave velocity. Vasc Health Risk Manag. 2018;14:41-62.

15. Ferreira MT, Leite NC, Cardoso CR, Salles GF. Correlates of aortic stiffness progression in patients with type 2 diabetes: importance of glycemic control: the Rio de Janeiro type 2 diabetes cohort study. Diabetes Care. 2015;38:897-904.

16. Yamashina A, Tomiyama H, Arai T, Hirose K, Koji Y, Hirayama Y, et al. Brachial-ankle pulse wave velocity as a marker of atherosclerotic vascular damage and cardiovascular risk. Hypertens Res. 2003;26:615-22.

17. Chen S, Li W, Jin C, Vaidya A, Gao J, Yang H, et al. Resting heart rate trajectory pattern predicts arterial stiffness in a community-based chinese cohort. Arterioscler Thromb Vasc Biol. 2017;37:359-64.

18. Zheng M, Zhang X, Chen S, Song Y, Zhao Q, Gao X, et al. Arterial stiffness preceding diabetes: a longitudinal study. Circ Res. 2020;127:1491-8.

19. Kundu S, Aulchenko YS, van Duijn CM, Janssens AC. PredictABEL: an R package for the assessment of risk prediction models. Eur J Epidemiol. 2011;26:261-4.

20. Polonis K, Hoffmann M, Szyndler A, Wolf J, Nowak R, Becari C, et al. A multilocus genetic risk score is associated with arterial stiffness in hypertensive patients: the CARE NORTH study. J Hypertens. 2018;36:1882-8.

21. Do R, Willer CJ, Schmidt EM, Sengupta S, Gao C, Peloso GM, et al. Common variants associated with plasma triglycerides and risk for coronary artery disease. Nat Genet. 2013;45:1345-52.

22. Yu KJ, Zhang JR, Li Y, Huang X, LiU T, Li C, et al. Gallstone disease is associated with arterial stiffness progression. Hypertens Res. 2017:40:31-4.

23. Li N, Zhang GW, Zhang JR, Jin D, Li Y, Liu T, et al. Non-alcoholic fatty liver disease is associated with progression of arterial stiffness. Nutr Metab Cardiovasc Dis. 2015;25:218-23.

24. Zachariah JP, Rong J, Larson MG, Hamburg NM, Benjamin EJ, Vasan RS, et al. Metabolic predictors of change in vascular function: prospective associations from a community-based cohort. Hypertension. 2018;71:237-42.

25. McEniery CM, Spratt M, Munnery M, Yarnell J, Lowe GD, Rumley A, et al. An analysis of prospective risk factors for aortic stiffness in men: 20-year follow-up from the Caerphilly prospective study. Hypertension. 2010;56:36-43.

26. Benetos A, Adamopoulos C, Bureau JM, Temmar M, Labat C, Bean K, et al. Determinants of accelerated progression of arterial stiffness in normotensive subjects and in treated hypertensive subjects over a 6-year period. Circulation. 2002;105:1202-7.

27. Utescu MS, Couture V, Mac-Way F, De Serres SA, Marquis K, Lariviere R, et al. Determinants of progression of aortic stiffness in hemodialysis patients: a prospective longitudinal study. Hypertension. 2013;62:154-60. 
28. Cecelja M, Jiang B, Keehn L, Hussain T, Silva Vieira M, Phinikaridou A, et al. Arterial stiffening is a heritable trait associated with arterial dilation but not wall thickening: a longitudinal study in the twins UK cohort. Eur Heart J. 2018;39:2282-8.

29. Tomiyama H, Hashimoto H, Tanaka H, Matsumoto C, Odaira M, Yamada J, et al. Continuous smoking and progression of arterial stiffening: a prospective study. J Am Coll Cardiol. 2010;55:1979-87.

30. McLaughlin T, Abbasi F, Cheal K, Chu J, Lamendola C, Reaven G. Use of metabolic markers to identify overweight individuals who are insulin resistant. Ann Intern Med. 2003;139:802-9.

31. Gasevic D, Frohlich J, Mancini GB, Lear SA. The association between triglyceride to high-density-lipoprotein cholesterol ratio and insulin resistance in a multiethnic primary prevention cohort. Metabolism. 2012;61:583-9.

32. Zheng D, Li H, Ai F, Sun F, Singh M, Cao X, et al. Association between the triglyceride to high-density lipoprotein cholesterol ratio and the risk of type 2 diabetes mellitus among Chinese elderly: the Beijing Longitudinal Study of Aging. 2020;8.

33. Edwards MK, Blaha MJ, Loprinzi PD. Atherogenic index of plasma and triglyceride/high-density lipoprotein cholesterol ratio predict mortality risk better than individual cholesterol risk factors, among an older adult population. Mayo Clin Proc. 2017;92:680-1.

34. Chen C, Dai JL. Triglyceride to high-density lipoprotein cholesterol (HDLC) ratio and arterial stiffness in Japanese population: a secondary analysis based on a cross-sectional study. Lipids Health Dis. 2018;17:130.

35. Chung TH, Shim JY, Kwon YJ, Lee YJ. High triglyceride to high-density lipoprotein cholesterol ratio and arterial stiffness in postmenopausal Korean women. 2019;21:399-404.

36. Poon AK, Meyer ML, Tanaka H, Selvin E, Pankow J, Zeng D, et al. Association of insulin resistance, from mid-life to late-life, with aortic stiffness in late-life: the Atherosclerosis Risk in Communities Study. Cardiovasc Diabetol. 2020;19:11.

37. Urbina EM, Khoury PR, McCoy CE, Dolan LM, Daniels SR, Kimball TR, Triglyceride to $\mathrm{HDL}-\mathrm{C}$ ratio and increased arterial stiffness in children, adolescents, and young adults. Pediatrics. 2013;131:e1082-1090.

38. Li G, Wu HK, Wu XW, Cao Z, Tu YC, Ma Y, et al. Small dense low density lipoprotein-cholesterol and cholesterol ratios to predict arterial stiffness progression in normotensive subjects over a 5-year period. Lipids Health Dis. 2018;17:27.
39. Humphrey JD, Harrison DG, Figueroa CA, Lacolley P, Laurent S. Central artery stiffness in hypertension and aging: a problem with cause and consequence. Circ Res. 2016;118:379-81.

40. Mitchell GF. Arterial stiffness and hypertension: chicken or egg? Hypertension. 2014;64:210-4

41. Nilsson PM, Boutouyrie P, Laurent S. Vascular aging: a tale of EVA and ADAM in cardiovascular risk assessment and prevention. Hypertension. 2009;54:3-10

42. Guo J, Fujiyoshi A, Willcox B, Choo J, Vishnu A, Hisamatsu T, et al. Increased aortic calcification is associated with arterial stiffness progression in multiethnic middle-aged men. Hypertension. 2017;69:102-8.

43. Wang M, Luo X, Xu S, Liu W, Ding F, Zhang X, et al. Trends in smoking prevalence and implication for chronic diseases in China: serial national cross-sectional surveys from 2003 to 2013. Lancet Respir Med. 2019;7:35-45.

44. Chelland Campbell S, Moffatt RJ, Stamford BA. Smoking and smoking cessation: the relationship between cardiovascular disease and lipoprotein metabolism: a review. Atherosclerosis. 2008;201:225-35.

45. Charakida M, Georgiopoulos G, Dangardt F, Chiesa ST, Hughes AD, Rapala $A$, et al. Early vascular damage from smoking and alcohol in teenage years: the ALSPAC study. Eur Heart J. 2019;40:345-53.

46. Price JF, Mowbray PI, Lee AJ, Rumley A, Lowe GD, Fowkes FG. Relationship between smoking and cardiovascular risk factors in the development of peripheral arterial disease and coronary artery disease: Edinburgh Artery Study. Eur Heart J. 1999;20:344-53.

47. Song W, Guan J, He P, Fan S, Zhi H, Wang L. Mediating effects of lipids on the association between smoking and coronary artery disease risk among Chinese. Lipids Health Dis. 2020;19:149.

48. Tang R, Yang S, Liu W, Yang B, Wang S, Yang Z, et al. Smoking is a risk factor of coronary heart disease through HDL-C in Chinese T2DM patients: a mediation analysis. J Healthc Eng. 2020;2020:8876812.

\section{Publisher's Note}

Springer Nature remains neutral with regard to jurisdictional claims in published maps and institutional affiliations.
Ready to submit your research? Choose BMC and benefit from:

- fast, convenient online submission

- thorough peer review by experienced researchers in your field

- rapid publication on acceptance

- support for research data, including large and complex data types

- gold Open Access which fosters wider collaboration and increased citations

- maximum visibility for your research: over $100 \mathrm{M}$ website views per year

At BMC, research is always in progress.

Learn more biomedcentral.com/submissions 